\title{
Probabilistic Seismic Analysis of the Deep Sliding Stability of a Concrete Gravity Dam-Foundation System
}

\author{
Hui Liang $\mathbb{D}^{1,2}$ Shengshan Guo ${ }^{1}{ }^{1,2}$ Yifu Tian, ${ }^{3}$ Jin Tu, ${ }^{1,2}$ Deyu Li, ${ }^{1,2}$ and Chunli Yan ${ }^{1,2}$ \\ ${ }^{1}$ State Key Laboratory of Simulation and Regulation of Water Cycle in River Basin, Beijing 100048, China \\ ${ }^{2}$ Earthquake Engineering Research Centre, China Institute of Water Resources and Hydropower Research, Beijing, China \\ ${ }^{3}$ North China University of Water Resources and Electric Power, Zhengzhou 450046, China \\ Correspondence should be addressed to Shengshan Guo; 305265331@qq.com
}

Received 30 August 2020; Revised 9 November 2020; Accepted 20 November 2020; Published 9 December 2020

Academic Editor: Jia-wen Zhou

Copyright (C) 2020 Hui Liang et al. This is an open access article distributed under the Creative Commons Attribution License, which permits unrestricted use, distribution, and reproduction in any medium, provided the original work is properly cited.

\begin{abstract}
There are various uncertainties in the design, construction, and operation of dams. These uncertainties have an important impact on the seismic response and seismic safety evaluation of concrete dams. In this research, a typical nonoverflow monolith of a concrete gravity dam is selected as a case study for the sliding stability analysis. Based on the analysis and demonstration of parameter sensitivity of friction coefficients and cohesion and their influence on the deep antisliding stability of the damfoundation system, the probabilistic seismic analysis of a gravity dam-foundation system is carried out through Monte Carlo analysis with a large sample number. Damage levels are defined based on the sliding instability failure mode along with the corresponding threshold values of the damage index. Thus, seismic fragility analysis is investigated, and seismic fragility curves are obtained for the vulnerability assessment under earthquake hazards. The overall seismic stability of the gravity dam is evaluated, which provides the basis for the seismic safety evaluation in the probabilistic framework.
\end{abstract}

\section{Introduction}

Gravity dams as critical infrastructure components play an inestimable role in the economic and social development of the country for flood prevention, hydropower generation, water transportation, irrigation, and water supply. Once the failure of the dams happens, it could lead to potential loss of life and huge economic losses. The lack of a comprehensive understanding of the geological conditions of the foundation has been the main cause of the failure of gravity dams [1]. Thus, the sliding stability of dam-foundation systems has been a critical issue in seismic design, especially for the antisliding stability in the underlying complex foundation.

Currently, seismic design of gravity dams is usually performed by conventional seismic analysis methods with a lot of simplifications and assumptions of the model in the Chinese safety code [2], while the results tend to be overconservative and could not accurately reflect the influence of various complicated factors in the actual engineering problems. Moreover, there are two kinds of uncertainty in seismic assessment of engineering structures: (a) aleatory uncertainty, resulting from inherent variability in the natural process, and (b) epistemic uncertainty, due to the lack of knowledge or information of the system that needs to be considered [3-5]. Gravity dams, as important infrastructures, also have various uncertainties. These uncertainties indeed show a great impact on the seismic response of the concrete gravity dams [6]. Thus, probabilistic and stochastic analyses are preferred for an adequate understanding of the expected range of structural response. Tekie and Ellingwood [7] used a probabilistic framework to model sources of uncertainty for developing fragilities of concrete gravity dams to assess their performance against seismic hazards with selected earthquake records. An earlier probabilistic analysis has been performed by taking concrete properties and artificial seismic excitation as random variables over the spatial domain [8]. An effective and powerful probabilistic method has been proposed for comprehensively evaluating seismic response including both sources of uncertainty combining the Monte Carlo simulation with incremental dynamic analysis (IDA) [9]. This method has been widely used for estimating the variability of seismic response of 
concrete buildings and bridges with reliable and significant achievements [10-13]. Several investigations have also been carried out for seismic fragility evaluation of concrete gravity dams in view of uncertainties. Seismic performance and various limit states of the dam were determined by IDA, and three damage indexes were proposed for effective estimation of the damage level of the dam [14]. Bernier et al. [15] improved the existing approaches by including the spatial variation of the angle of friction to evaluate the seismic fragility of a concrete dam. In research, IDA is used to quantify the damage levels and investigate the relationship of seismic intensity measures and the accumulation of damage on concrete gravity dams in consideration of record-torecord uncertainty [16]. The seismic performance sensitivity and uncertainty analysis of gravity dams is performed by taking the main characteristics of the nonlinear tensile behavior and seismic intensity measure as random variables [17]. In our previous study, seismic stability sensitivity and uncertainty analysis of a high arch dam-foundation system is performed by choosing the slippage and sliding area ratio as engineering demand parameters (EDPs), and the difference that lies in the two EDPs for evaluating the seismic performance of the arch dam is discussed [18].

Nevertheless, attempts have been partly made on quantitative evaluation of the impact of uncertainties on the deep antisliding stability of concrete gravity dams. Moreover, the effect of parameter variation on the ultimate instability and seismic fragility analysis of gravity dams requires additional assessment. Therefore, this paper focuses on the effects of parameter sensitivity and uncertainty and seismic fragility analysis of concrete gravity dams based on the sliding instability failure mode on account of the complexity of engineering geological conditions and lack of clear understanding of geological defects in the underlying dam foundation. The viscoelastic artificial boundary model is used for numerical simulation of infinite foundations. The dynamic contact force model is adopted to solve the nonlinearity contact problems in antisling stability of concrete gravity dams. The friction coefficients and cohesion [19] are chosen as random modeling parameters. By taking the typical nonoverflow monolith of a gravity dam foundation as a case study, the parameter sensitivity analysis is performed first to quantitatively understand the effect of each parameter on the seismic response of the concrete gravity dam. Then, the probabilistic analysis is carried out to propagate the sliding parameter uncertainties to the IDA-evaluated seismic performance with efficient Monte Carlo simulation and moment estimation techniques. Following the results of probabilistic analysis, damage levels are defined, and seismic fragility curves are obtained for the failure probability estimation of the sliding stability under earthquake hazards.

\section{Numerical Modeling}

2.1. Dam Description. The concrete gravity dam selected is a typical nonoverflow monolith of a gravity dam. There are 30 small faults in the dam site, and the developmental characteristics of the joint fissures show an obvious difference in tectonic location. Engineering geological conditions of the dam foundation are very unfavorable for the sliding stability. A number of weak interlayers and internal sedimentary bed downstream of the dam foundation and a large number of weak interlayers are found. The combination of all these unfavorable factors constitutes a geological background in which the concrete gravity dam may have sliding instability.

The geometric properties of the cross section of the dam and the sliding block are described in Figures 1(a) and 1(b). The concrete gravity dam rises $162 \mathrm{~m}$ above the base from El. $222 \mathrm{~m}$ to El. $384 \mathrm{~m}$. The width of the dam at the crest is $12 \mathrm{~m}$ and $168.5 \mathrm{~m}$ at the base. It is sloped at $1.0 \mathrm{~V}: 0.75 \mathrm{H}$ in the downstream area and $1 \mathrm{~V}: 0.2 \mathrm{H}$ in the upstream area. According to geological data, the angle between the upstream sliding surface (main sliding surface) and the horizontal direction is $18.7^{\circ}$. There is no clear exposed sliding surface in the downstream area, and the angle between the downstream sliding surface (auxiliary sliding surface) and the horizontal direction is obtained as $47.11^{\circ}$ according to the principle of the minimum safety factor.

2.2. Model Configuration. The finite element model which reflects the layout of the dam and actual geological conditions is established, shown in Figure 1(c).

The radiation damping effect and the far-field of the semi-infinite foundation are considered by the application of the viscoelastic artificial boundary model. The hydrodynamic pressure of the reservoir is simulated by Westergaard's added mass method [20] regardless of the compressibility of the reservoir. Double oblique sliding surfaces between the dam and foundation including the main sliding surface and auxiliary sliding surface are considered for the sliding stability analysis. The dynamic contact force model is adopted to solve the contact nonlinearity. In view of the fact that the resistant force at sliding surfaces is supposed to be controlled exclusively by friction coefficients and cohesion, friction coefficients and cohesion are taken as random variables, represented by $\mu_{1}, c_{1}$ and $\mu_{2}, c_{2}$ in the present study. The "base-case" physical mechanic material properties for the dam concrete and foundation are listed in Table 1.

The static load is composed of the upstream normal water level pressure, downstream water pressure, self-gravity of the dam body, upstream sediment pressure, and uplift pressure on the dam-foundation. Upstream and downstream normal water levels are $380.00 \mathrm{~m}$ and $265.80 \mathrm{~m}$, respectively. The upstream sediment elevation is $310 \mathrm{~m}$. The bulk density and internal friction angle are $7.8 \mathrm{kN} / \mathrm{m}^{3}$ and $20^{\circ}$.

\section{Seismic Sliding Stability Assessment}

Incremental dynamic analysis (IDA) [9] is a powerful and practical parametric analysis method that can provide a comprehensive evaluation of the structural performance under seismic loads. A suite of dynamic analyses of a structure suffering from one (or more) ground motion(s), each scaled to multiple increasing levels of seismic intensity, is required to implement IDA. Thereby, IDA curves of a 


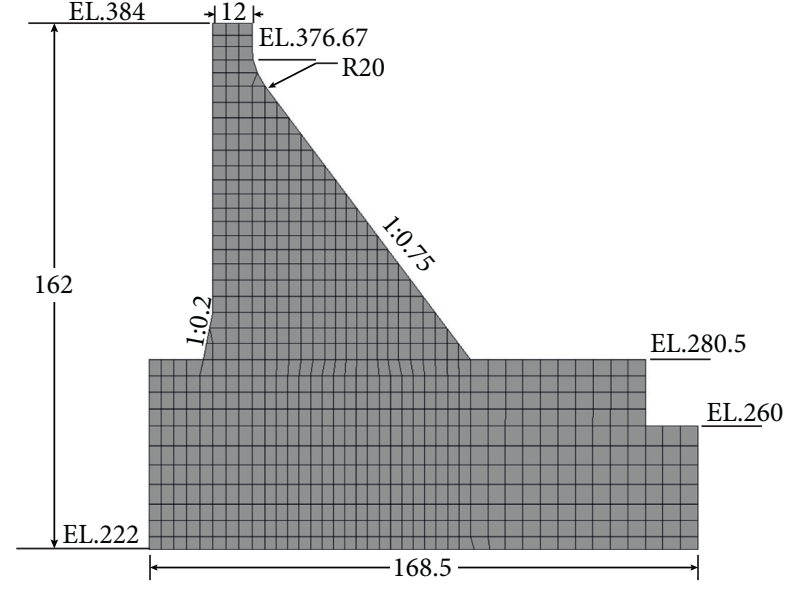

(a)

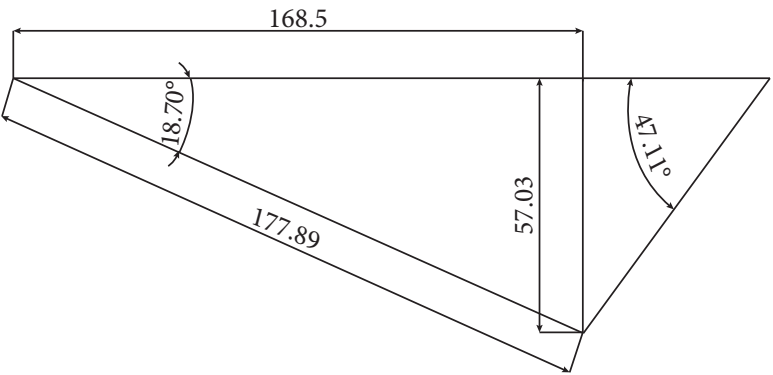

(b)

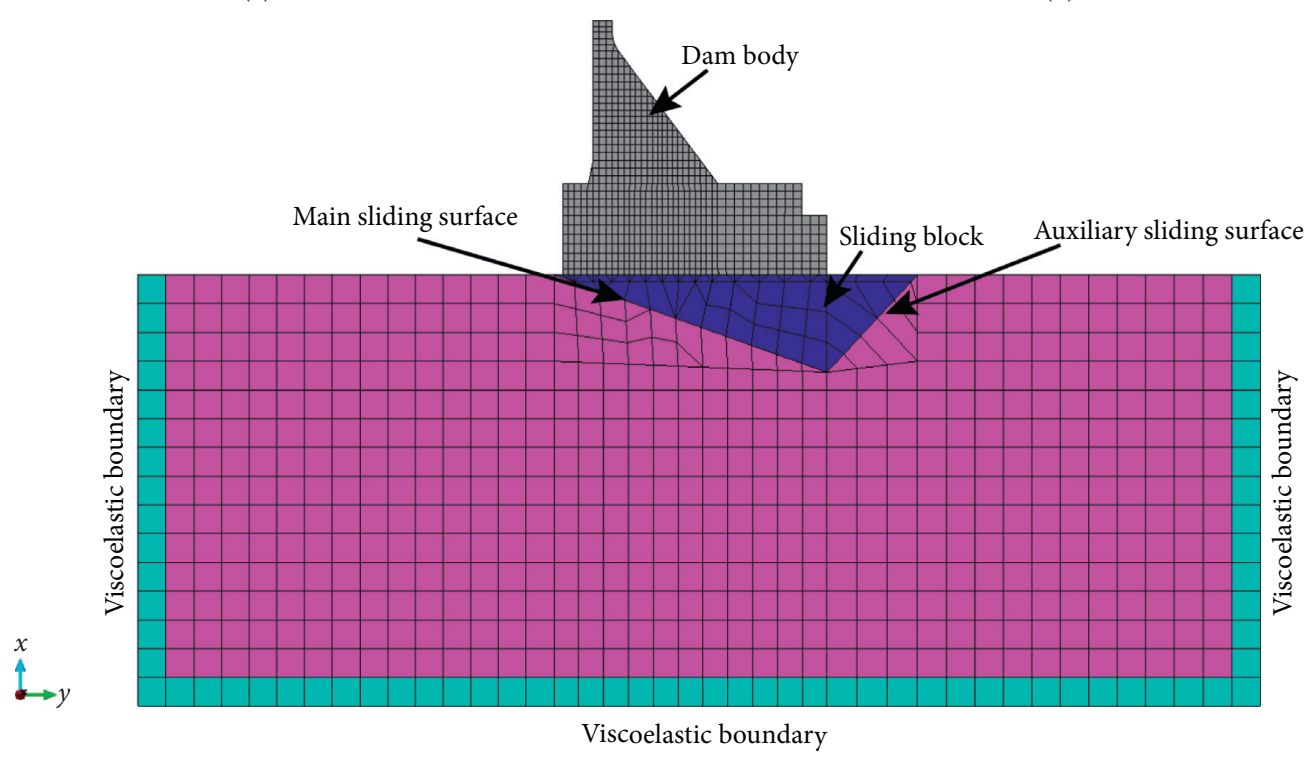

(c)

Figure 1: (a) Description of the typical nonoverflow monolith of the gravity dam (all dimensions are in meters). (b) Description of the sliding block. (c) Finite element mesh of the dam with the component of dam body, slide block, and truncated boundaries of foundation.

TABle 1: "Base-case" material properties for concrete and foundation.

\begin{tabular}{lccc}
\hline Material parameters & Symbol & unit & Value \\
\hline Concrete mass density & $\rho_{c}$ & $\mathrm{~kg} / \mathrm{m}^{3}$ & 2400 \\
Concrete elastic modulus & $\mathrm{E}_{c}$ & $\mathrm{GPa}$ & 26 \\
Concrete Poisson's ratio & $v_{c}$ & - & 0.167 \\
Foundation mass density & $\rho_{f}$ & $\mathrm{~kg} / \mathrm{m}^{3}$ & 2550 \\
Foundation deformation modulus & $\mathrm{E}_{f}$ & $\mathrm{GPa}$ & 13 \\
Foundation Poisson's ratio & $v_{f}$ & - & 0.25 \\
Friction coefficient of the main sliding surface & $\mu_{1}$ & - & 0.4 \\
Cohesion of main sliding surface & $c_{1}$ & $\mathrm{MPa}$ & 0.3 \\
Friction coefficient of the auxiliary sliding surface & $\mu_{2}$ & - & 1.0 \\
Cohesion of the auxiliary sliding surface & $c_{2}$ & $\mathrm{MPa}$ & 0.9 \\
\hline
\end{tabular}

seismic intensity measure (IM) versus a damage measure (DM) or engineering demand parameter (EDP) are obtained. Besides, the seismic capacity of a structure can be determined, and its limit states (LSs) are defined through IDA curves. For IM, the 5\% damping spectral acceleration at the fundamental vibration period of the base-case model $\left(S_{a}\right.$ $\left(T_{1}^{\text {base }}, 5 \%\right)$ ) and the peak ground acceleration (PGA) has been shown to be the two most widely used by most researchers $[10,15,21]$. For the sake of simplicity and convenience, PGA is chosen as IM in the seismic analysis. The slippage of the starting point on the main sliding surface and the ending point on the auxiliary sliding surface which play a critical role to assess the sliding stability is selected as EDP.

Based on ground motion parameters approved by seismological departments in China, considering the safety and simplified calculation, the site-related design response spectrum corresponding to the exceedance probability of $2 \%$ in 100 years is taken as the target spectrum to produce the artificial ground motions, which are normalized and shown in Figure 2. The PGA of vertical direction is two-thirds of that of the stream in the analysis. The PGA is scaled to multiple levels from zero to $1.0 \mathrm{~g}$ with steps of $0.1 \mathrm{~g}$ for the purpose of accurately reflecting the shearing slide of the 
contact surface in the IDA. An IDA curve is the plot of IM versus EDP for each model under each scaled ground motion and is generated from discrete points with spline interpolation.

Thus, the IDA method is adopted to investigate the parameter sensitivity and uncertainty of the friction coefficients and cohesion. Moreover, seismic fragility analysis of the concrete gravity dam-foundation system is performed based on the deep sliding failure mode.

\section{Evaluation of Parameter Sensitivity}

The sensitive analysis is applied to evaluate the sensitivity of each parameter for the following pairs of modifications: $\mu_{1}=\{0.2,0.6\}, c_{1}(\mathrm{MPa})=\{0.04,1.12\}, \mu_{2}=\{0.6,1.4\}$, and $c_{2}$ $(\mathrm{MPa})=\{0.43,1.68\}$.

4.1. Sensitivity of Friction Coefficients. The sensitivity of friction coefficients is discussed first. The shearing slides of the starting point on the main sliding surface and the ending point on the auxiliary sliding surface versus PGA are shown in Figures 3(a)-3(d), where the base-case model is plotted in a solid one.

Figures 3(a) and 3(b) depict the effect of friction coefficient $\mu_{1}$ on the shearing slid. From Figure 3(a), compared with the base-case model $\left(\mu_{1}=0.4\right)$, increasing $\mu_{1}$ by $50 \%$ greatly decreases the shearing slide of the starting point, and with the increase of PGA, the influence is weakened. As for the circumstance of decreasing $\mu_{1}$ by $50 \%$, it shows a totally opposite pattern. Figure 3(b) shows that the variation of $\mu_{1}$ plays a slight influence on the shearing slide of the ending point.

Figures 3(c) and 3(d) describe the effect of the friction coefficient $\mu_{2}$ on the shearing slide. Increasing or decreasing the friction coefficient $\mu_{2}$ shows nearly no effect on the shear slide before the occurrence of the inflection points. While the inflection point appears, the IDA curves start growing rapidly and the effect becomes evident. Increasing $\mu_{2}$ by $40 \%$ reduces the shearing slide with PGA ranging from $0.6 \mathrm{~g}$ to $1.0 \mathrm{~g}$, which represents the enhancement of the sliding stability of the dam. Otherwise, lowing $\mu_{2}$ to 0.6 has an obviously opposite effect.

In conclusion, the deep antisliding stability of the gravity dam is sensitive to friction coefficients, which is consistent with the results obtained in [18]. The friction coefficient $\mu_{1}$ plays a leading role in the deep sliding stability of the main sliding surface, and the friction coefficient $\mu_{2}$ plays a dominant role in the overall sliding stability of the gravity dam.

4.2. Sensitivity of Cohesion. Figures 4(a)-4(d) clearly show the sensitivity of sliding stability to cohesion, and the solid line represents the base-case model with the cohesion of $c_{1}=0.30 \mathrm{MPa}, c_{2}=0.90 \mathrm{MPa}$.

From Figures $4(a)-4(d)$, the variation of cohesion has little effect on the shearing slides. A relatively distinct difference appears at PGA of $0.1 \mathrm{~g}$ in Figure 4(a). The reason is that larger cohesion $c_{1}$ can provide sufficient sliding resistance force to prevent the generation of initial sliding on the main sliding surface under small seismic intensity levels. It is notable that the cohesion has a great influence on the occurrence of initial sliding and a slight effect on the development of the slippage.

\section{Probabilistic Analysis}

Based on the sensitivity analysis above, parameter uncertainties do exist in the sliding stability of the concrete gravity dam. However, these uncertainties are difficult to be evaluated and predicted by deterministic models. Therefore, the probabilistic analysis is performed to examine the effects of parameter uncertainties first. Afterward, the seismic fragility analysis is carried out for the failure probability estimation of the dam under different seismic intensity levels. The friction coefficients and cohesion are considered as random variables. It is difficult to estimate the probability distributions of the random variables due to the lack of knowledge or limited information. The most relevant literature $[13,17,22]$ appropriately defines the probability distributions of random parameters artificially. However, for the sliding stability of concrete gravity dams, probability distributions of friction coefficients and cohesion are clearly pointed out to be normal distribution and lognormal distribution, respectively [23]. The distribution of each parameter is truncated with a reasonable minimum and maximum to meet with physical limits. The hard limits set at 2 standard deviations away from the central value are considered to cover most of the samples. Thus, the distributions are assigned independently to each parameter with a mean consistent with the base-case model, shown in Table 2.

5.1. Latin Hypercube Sampling. In the probabilistic seismic analysis of structures, random variables need to be sampled. Samples of random variables are input into the model as the deterministic parameters to carry out the finite element analyses, which are computationally expensive. In order to reduce the computational effort, it is necessary to choose an appropriate sampling method. At present, the main sampling methods are random sampling, stratified sampling, and Latin hypercube sampling (LHS), etc. LHS, a multilevel stratified sampling method with a widely used application, provides an efficient way of sampling variables from their distributions [24].

Herein, LHS is adopted for random sampling of the fiction coefficients and cohesion at the dam-foundation contact. Based on the assumption that the variables are independent of each other, the procedure of LHS can be summarized as follows: (1) divide the probability distribution of each variable into $N$ equal-probable intervals; (2) select a value randomly from each interval; for the $i$ th interval, the sampled cumulative probability can be written as $\operatorname{Prob}_{i}(1 / N) r_{u}+(i-1) / N$, where $r_{u}$ is uniformly distributed random number ranging from 0 to 1 ; (3) transform the probability values sampled into the value $x$ using the inverse of probability distribution function $F^{-1}$ with $x=F^{-1}$ (Prob); (4) the $N$ values obtained for each variable $x$ are paired randomly. However, there are no specific standards for 


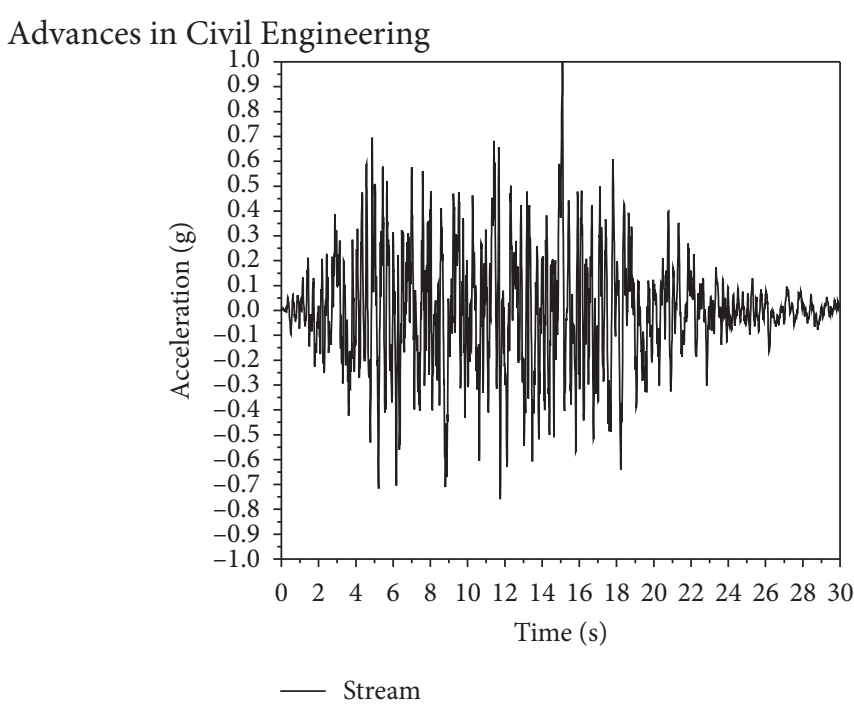

(a)

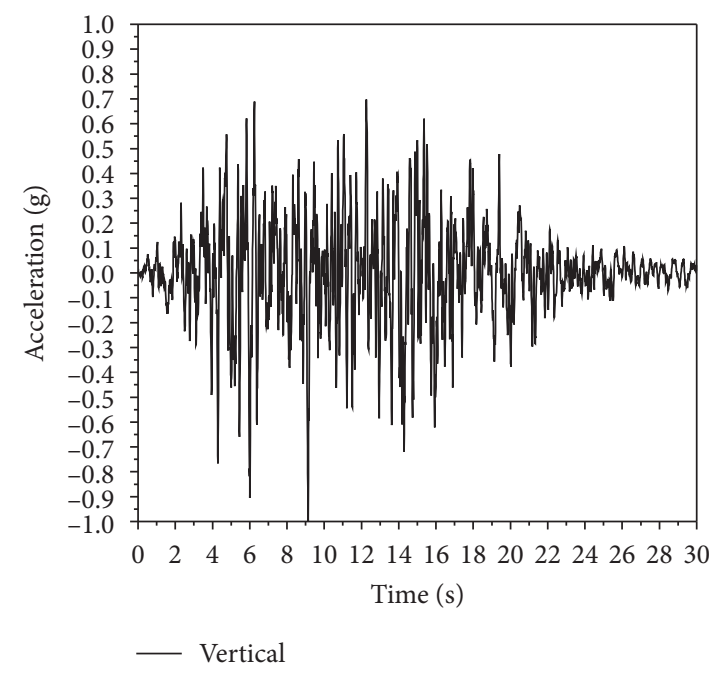

(b)

FIgURE 2: The normalized artificial acceleration time histories.

determining the sample size $N$ to estimate uncertainties of the parameters accurately. A total of 20 samples for each uncertain parameter are used [15], and the sample sizes $N$ of 30 and 200 are obtained by LHS separately [13, 17]. Therefore, taking into account the number of simulations and reliability of the results, the sample size $N$ is taken as 100 to ensure the accuracy of the probabilistic analysis to evaluate the parameter uncertainties.

Research on parameter uncertainties is carried out by performing IDA for each sample. The total number of simulations is 1000 with intensity levels of 10 being multiplied by samples of 100 . The IDA curves with their $16 \%$, $50 \%$, and $84 \%$ fractile are shown in Figure 5.

Figure 5(a) shows the shearing slide on the main sliding surface influenced by the variation of the parameters. It can be divided into three stages as follows: (1) the obvious impact before PGA at $0.3 \mathrm{~g}$, (2) minor influence of PGA between $0.3 \mathrm{~g}$ and $0.6 \mathrm{~g}$, and (3) high variability after PGA of $0.6 \mathrm{~g}$. The variability of the shearing slide on the auxiliary sliding surface is shown in Figure 5(b). Clearly, the shearing slide generates and gradually increases until PGA is about $0.3 \mathrm{~g}$. As the obvious shearing slide on the auxiliary sliding surface occurs until PGA comes to $0.6 \mathrm{~g}$, the variability tends to be inconspicuous before PGA of $0.6 \mathrm{~g}$.

According to the discussions above, a uniform conclusion can be obtained: the prominent influence of parameter uncertainties on the sliding stability of the concrete gravity dam usually happens during the rapid development of the shearing slide. Meanwhile, it seems that the occurrence of turning points on both the main sliding surface and the auxiliary sliding surface varies from $0.5 \mathrm{~g}$ to $0.65 \mathrm{~g}$, which indicates the diversity of the antisliding instability of the concrete gravity dam caused by parameter uncertainties.

5.2. Median and Dispersion. In order to obtain more useful and convincing conclusions from the above parameter uncertainty analyses, it is necessary to quantitatively describe the probabilistic nature of the curves by evaluating their moments. Herein, the median and dispersion of the shearing slide at each intensity level of PGA are calculated. The median at each PGA level is defined as a central value, and the dispersion caused by the various random parameters to the central value is represented by its $\beta$-value, which is the standard deviation of the natural logarithm of the shearing slides conditioned on PGA. It needs to be informed that the only variability is epistemic uncertainty caused by parameters of the dam foundation, and aleatory uncertainty (record-to-record uncertainty) is beyond consideration in this paper.

Assuming that $\ln \Delta^{i}$ is the natural logarithm of the shearing slide for a given PGA, superscript $i(i=1, \ldots, N)$ is the $i$ th sample model. AVG ${ }_{l n} \Delta$ is the mean of the corresponding natural logarithm of the demand responses (shearing slide in this paper) over all realizations. The central value, $C_{\Delta}$, and its dispersion, $\beta_{U}$, due to parameter uncertainty can be obtained as

$$
\begin{aligned}
& C_{\Delta}=\operatorname{med}\left(\Delta^{i}\right), \\
& \beta_{U}=\sqrt{\frac{\sum_{i=1}^{N}\left(\ln \Delta^{i}-\mathrm{AVG}_{\ln \Delta}\right)^{2}}{N-1},}
\end{aligned}
$$

where "med" is the median operator for the overall random samples.

The results of the overall mean, median (50\% fractile), and base-case model IDA curves conditioned on PGA are shown in Figure 6. It is remarkable that the overall mean and median of IDA curves with LHS show excellent consistency with the base-case model for both the starting point and ending point at each intensity level. Thus, the overall median/mean capacity of the concrete gravity dam is basically equal to the capacity of the median/mean model. On the other hand, it would be advisable that one can use the basecase or median/mean model to accurately estimate the median/mean response of dam samples instead of performing computer-intensive IDA with LHS without confusion. 

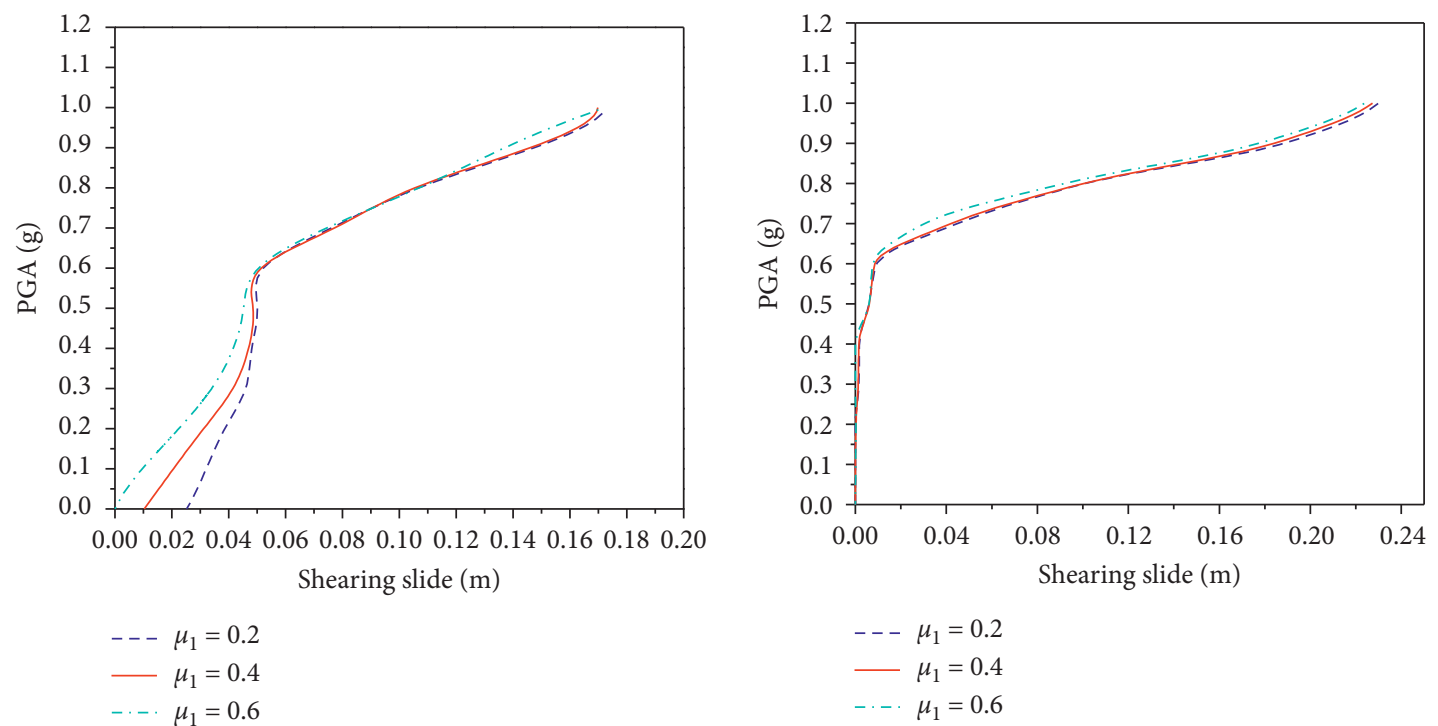

(a)

(b)

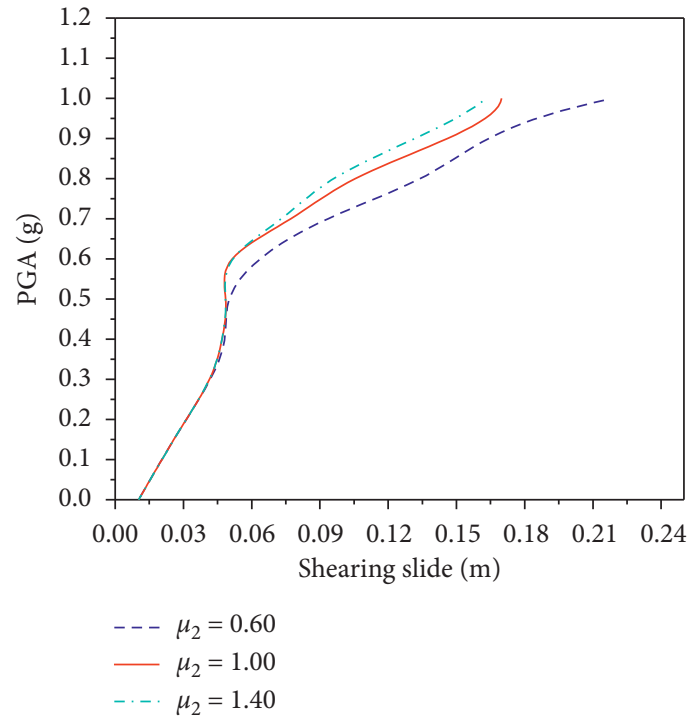

(c)

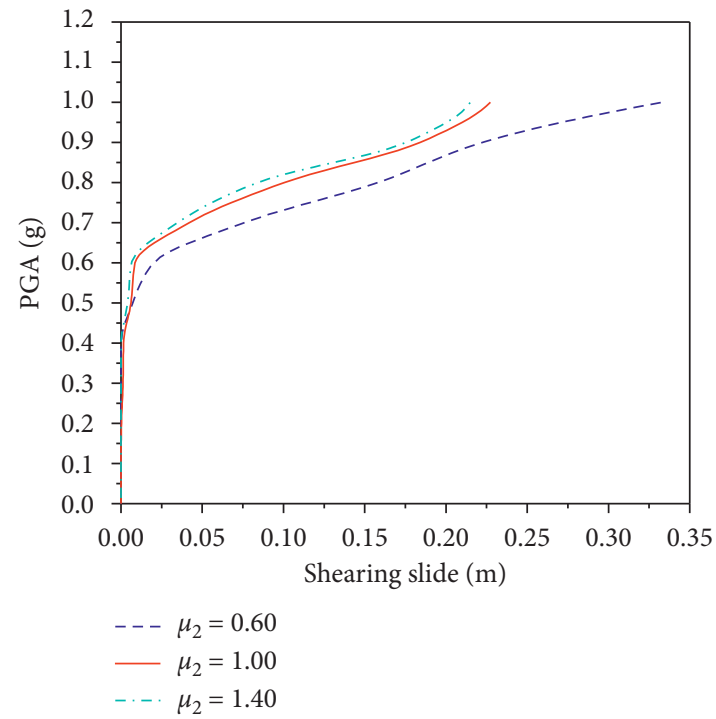

(d)

Figure 3: Sensitivity of IDA curves to friction coefficients. (a) $\mu_{1}$ affects the shearing slide of the starting point on the main sliding surface. (b) Minor effect of $\mu_{1}$ on the shearing slide of the ending point on the auxiliary sliding surface. (c) $\mu_{2}$ obviously affects the shearing slide of the starting point on the main sliding surface. (d) Distinct impact of $\mu_{2}$ on the shearing slide of the ending point on the auxiliary sliding surface.

TABLE 2: The probability distributions for the random parameters.

\begin{tabular}{lccccc}
\hline Material parameters & Mean & St. deviation & Minimum & Maximum & Type \\
\hline Friction coefficient $\mu_{1}$ & 0.40 & 0.100 & 0.20 & 0.60 & Normal \\
Cohesion $c_{1}(\mathrm{MPa})$ & 0.30 & 0.300 & 0.04 & 1.12 & Lognormal \\
Friction coefficient $\mu_{2}$ & 1.00 & 0.200 & 0.60 & 1.40 & Normal \\
Cohesion $c_{2}(\mathrm{MPa})$ & 0.90 & 0.315 & 0.43 & 1.68 & Lognormal \\
\hline
\end{tabular}

The evaluation of the parameters' dispersions, $\beta_{U}$, of the shearing slides of the starting point and ending point at each intensity level is shown in Figure 7(a). The parameter uncertainties of the starting point tend to be relatively smooth in comparison with those of the ending point in the entire intensity range. The maximum dispersion of the starting point appears at PGA of $0.1 \mathrm{~g}$, and it decreases rapidly with the increasing PGA levels ranging from $0.1 \mathrm{~g}$ to $0.3 \mathrm{~g}$. Afterward, it smoothly changes between the intensity range from $0.3 \mathrm{~g}$ to $0.6 \mathrm{~g}$, while it is noticeable that the dispersion of the ending point has a zero value at low intensity levels because of the slight shearing slide on the auxiliary sliding 

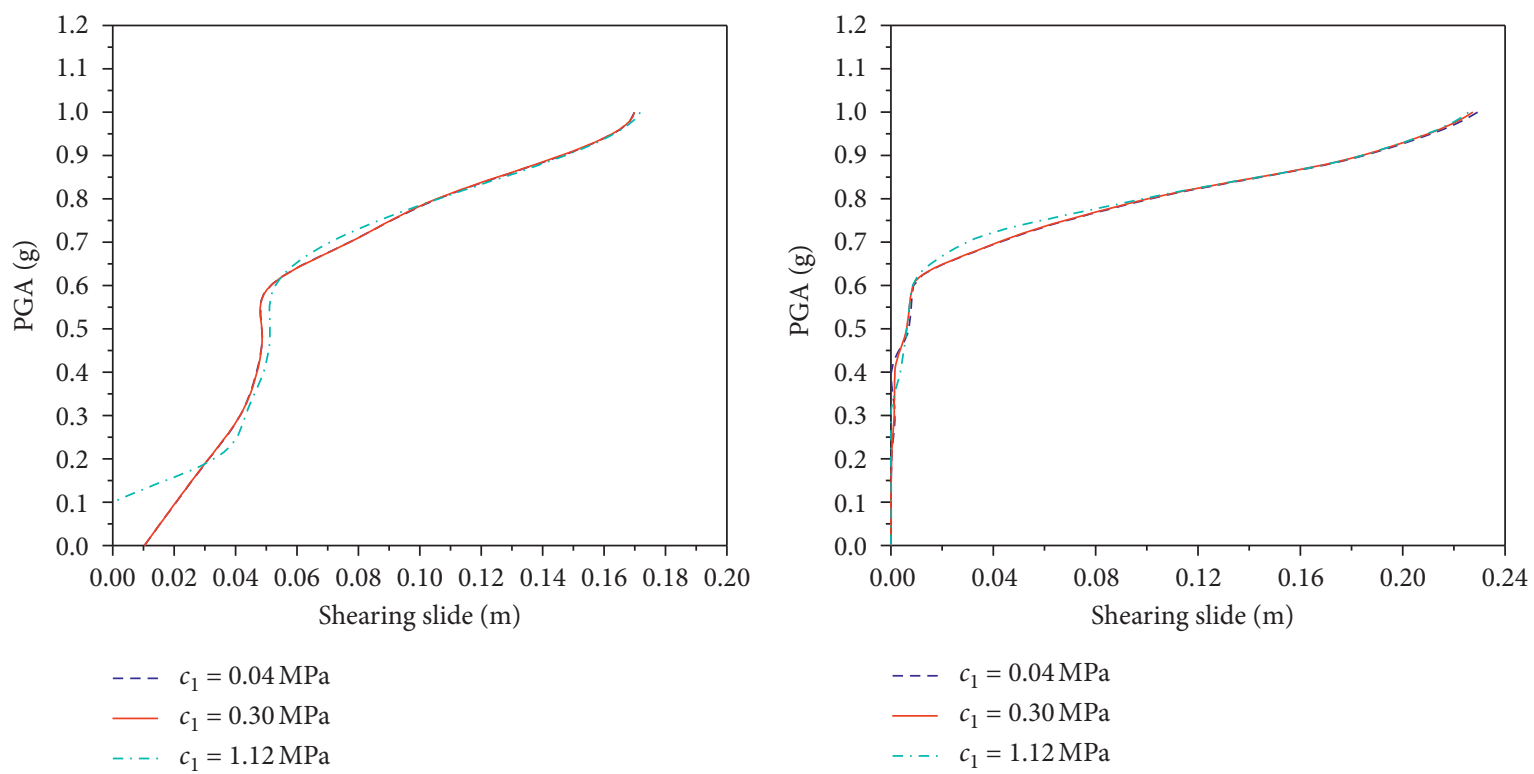

(a)
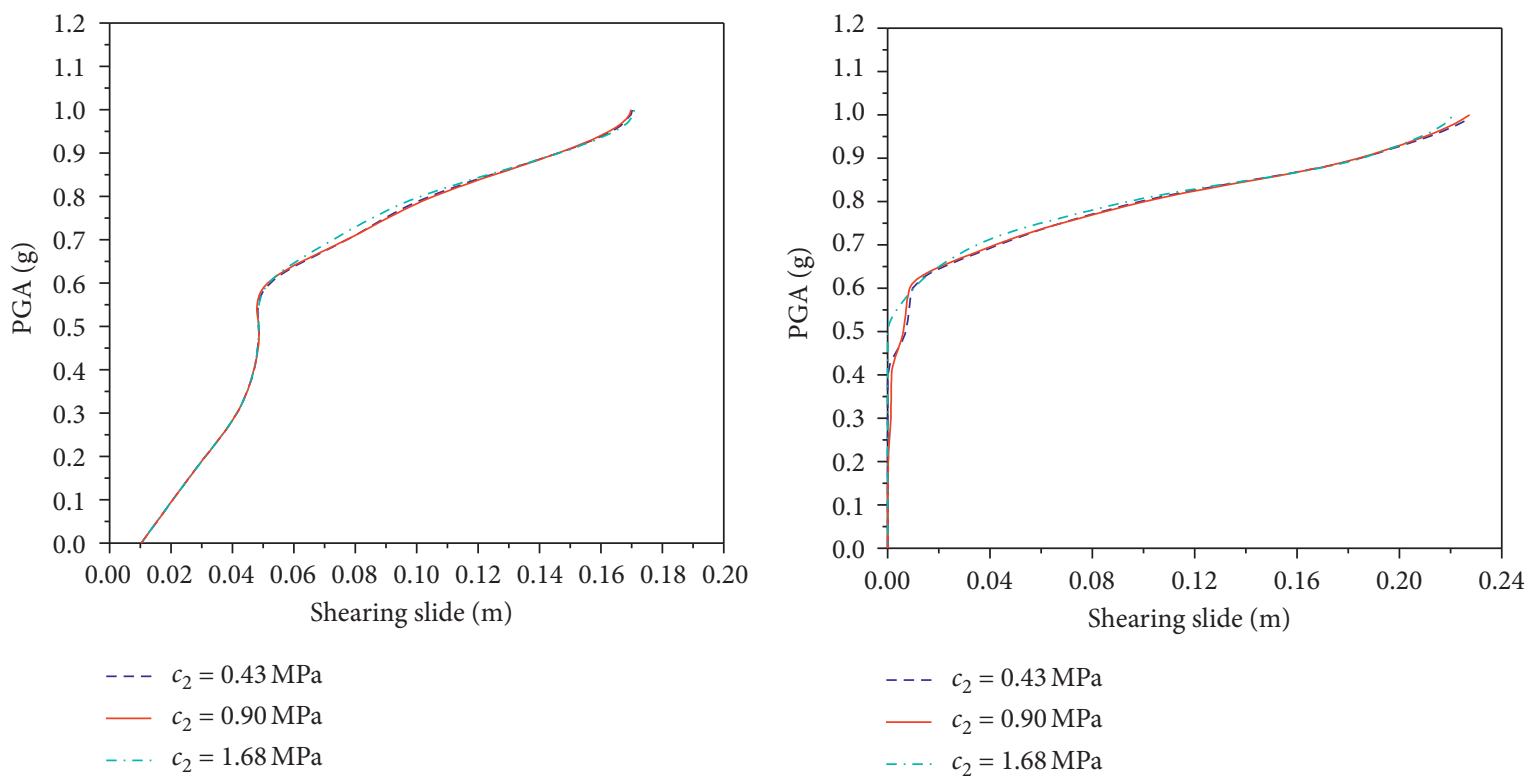

(c)

(d)

FIGURE 4: Sensitivity of IDA curves to cohesion: (a) $c_{1}$ affects the shearing slide of the starting point on the main sliding surface; (b) $c_{1}$ slightly affects the shearing slide of the ending point on the auxiliary sliding surface; (c) little effect of $c_{2}$ on the shearing slide of the starting point on the main sliding surface; (d) $c_{2}$ is not influential on the shearing slide of the ending point on the auxiliary sliding surface.

surface as discussed above. A marked increase appears at PGA of $0.6 \mathrm{~g}$ on account of the obvious sliding on the auxiliary sliding surface. Then, the dispersion experiences a rapid decline with the increase of PGA. The low dispersion of the results shows a good correlation of the response.

\subsection{Seismic Fragility Analysis}

5.3.1. Analytical Seismic Fragility Function. The seismic fragility curve provides a quantitative description of the probability of exceeding a certain level of failure of a system under seismic load. A lognormal cumulative distribution function is usually used to define a fragility function:

$$
P(\operatorname{LS} \mid \mathrm{IM}=\mathrm{im})=\Phi\left(\frac{\ln \mathrm{im}-\eta_{\mathrm{im}}}{\beta_{\mathrm{im}}}\right),
$$

where $P(\mathrm{LS} \mid \mathrm{IM}=\mathrm{im})$ equals the probability of the collapse of the structure under a ground motion with an intensity level, im; im is the ground motion intensity measure; $\eta_{\mathrm{im}}$ and $\beta_{\mathrm{im}}$ are the logarithmic mean value and logarithmic standard deviation of the IM values corresponding to each 


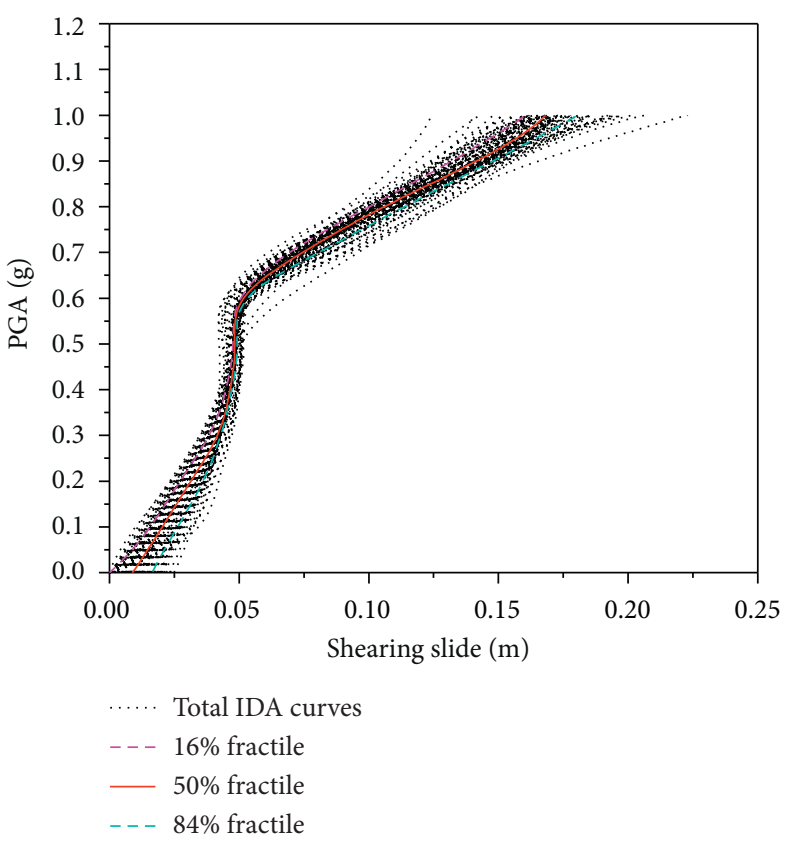

(a)

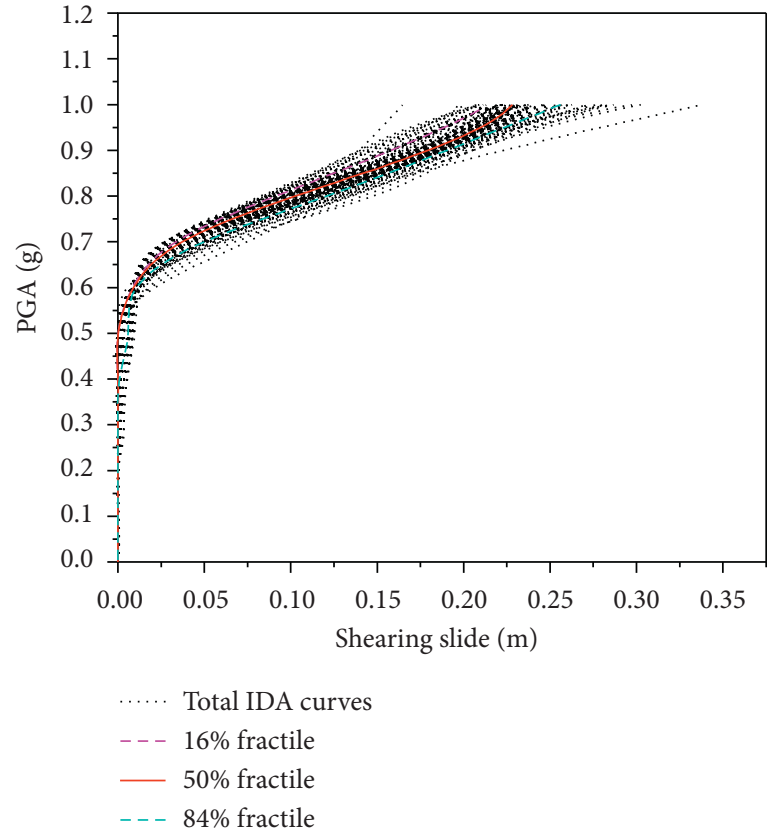

(b)

FIgURE 5: The total IDA curves with their $16 \%, 50 \%$, and $84 \%$ fractile of dam samples of (a) the shearing slide of the starting point on the main sliding surface and (b) the shearing slide of the ending point on the auxiliary sliding surface.

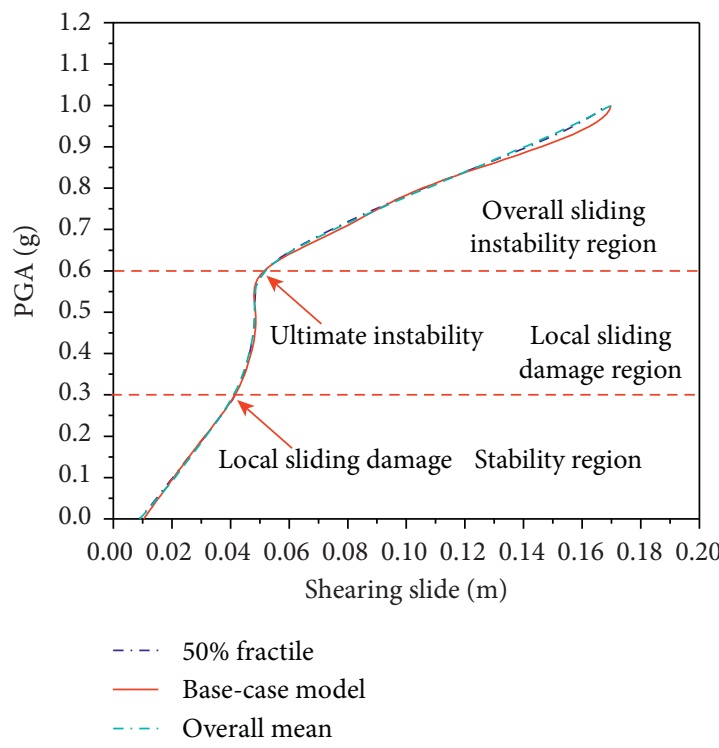

(a)

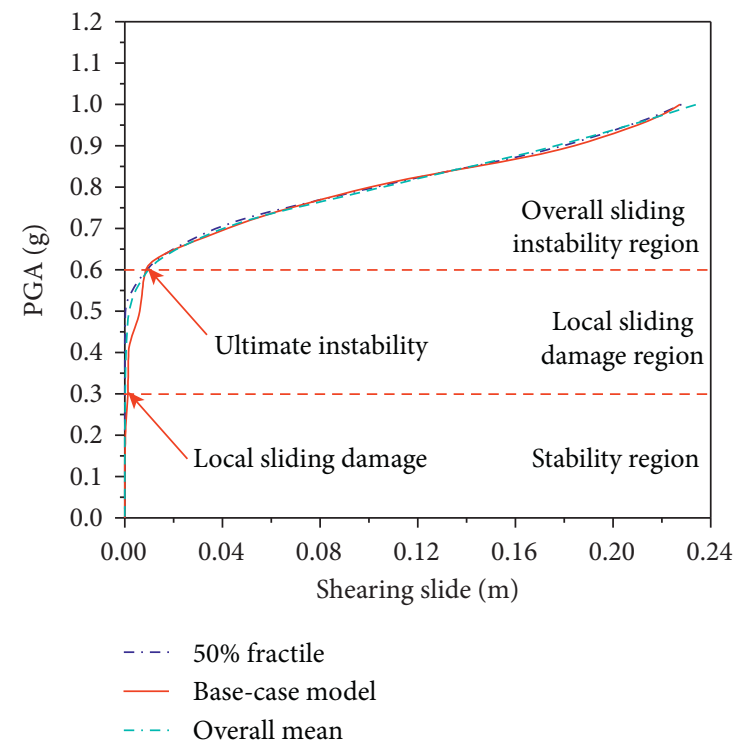

(b)

Figure 6: Median along with the base-case and overall mean shearing slides of (a) the starting point on the main sliding surface and (b) the ending point on the auxiliary sliding surface.

performance level; $\Phi(\cdot)$ represents the standard normal cumulative distribution function.

5.3.2. Limit States. The mean/median of the IDA curves is reasonable and usable for the determination of the capacity and limit states of structures [14]. As shown in Figures 6(a) and 6(b), the development process of the deep sliding stability of the gravity dam can be divided into three regions: (1) stability region, (2) local sliding damage region, and (3) overall sliding instability region. The limit states, related to structural failure modes, can be of significance for concrete gravity dams. Several damage levels are used considering sliding at the dam-foundation interface at the base of the 


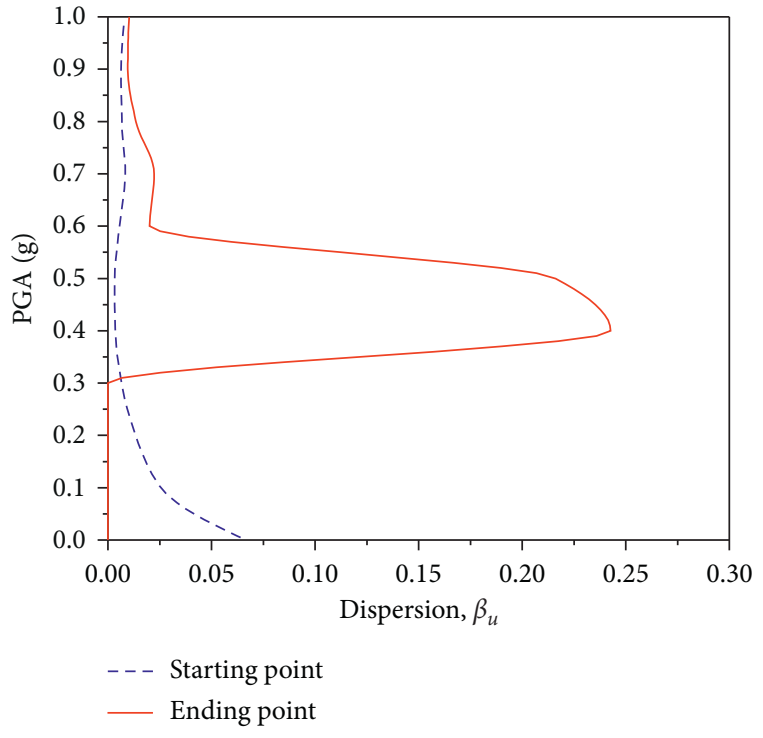

(a)

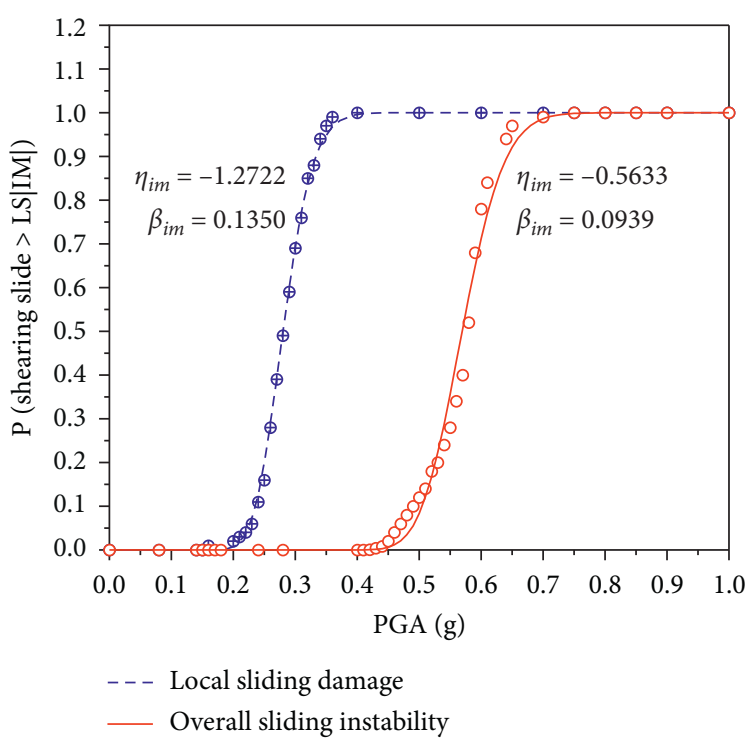

(b)

Figure 7: (a) Parameters' dispersion of the results of LHS of the starting point on the main sliding surface and the ending point on the auxiliary sliding surface. (b) Seismic fragility curves for the concrete gravity dam.

dam $[7,15]$. The situation that curtain safety and damage of the property of the drainage system are caused by interface dislocation should be of consideration. Shen [21] proposed preliminary suggestions for performance-based safety evaluation indexes on account of yield ratio of dam-foundation interface and performance of the drainage system. Thus, following the results of the previous analyses and performance evaluation indicators given by other scholars, damage levels based on the deep sliding failure mode can be defined as follows: (1) local sliding damage with the shearing slide of $40 \mathrm{~mm}$ on the main sliding surface and the shearing slide of less than $40 \%$ drainage hole diameter and (2) overall sliding instability corresponding to the overall sliding of the concrete gravity dam.

5.3.3. Seismic Fragility Curves. Based on the seismic fragility function and defined damage levels, seismic fragility curves are obtained in Figure 7(b). The results of the seismic fragility analysis offer insights into the vulnerability of the dam and the risk of damage under different earthquake hazards. For instance, as the PGA reaches $0.226 \mathrm{~g}$ (MDE, a return period criterion of 0.02 exceedance probability in 100 years), the probability of the occurrence of local sliding damage is less than $10 \%$, and the probability of reaching overall sliding instability is almost zero. Even the PGA comes to $0.282 \mathrm{~g}$ (MCE, a return period criterion of 0.01 exceedance probability in 100 years), the probability of the occurrence of local sliding damage is about $40 \%$ and that of the overall sliding failure is 0 indicating that the concrete gravity dam is able to maintain good deep sliding stability. A low probability of the overall sliding instability preliminarily happens at PGA of $0.4 \mathrm{~g}$, while the local sliding damage appears with the probability of about $100 \%$. When PGA reaches $0.65 \mathrm{~g}$, the probability of the overall sliding instability of the dam is $100 \%$.

\section{Conclusions}

The parameter sensitivity and uncertainty analysis and the seismic fragility analysis of a concrete gravity dam-foundation system are performed. The viscoelastic boundary method is adopted for the numerical simulation of the infinite foundation. The dynamic contact force model with the Lagrangian multiplier method for the contact nonlinearity controlled by friction coefficients and cohesion is adopted.

The sensitivity and uncertainty analysis is performed by taking friction coefficients and cohesion as random parameters. Results show that the friction coefficients obviously affect the shearing slide. The cohesion has a great influence on the occurrence of initial sliding on the main sliding surface and a slight effect on the development of the shearing slide.

The influence of the parameter uncertainties on the shearing slide of the main sliding surface can be divided into three stages: (1) the obvious impact before PGA at $0.3 \mathrm{~g}$, (2) minor influence of PGA between $0.3 \mathrm{~g}$ and $0.6 \mathrm{~g}$, and (3) high variability after PGA of $0.6 \mathrm{~g}$. It is remarkable that the overall median/mean capacity of the concrete gravity dam is basically equal to the capacity of the median/mean model. The low dispersion shows a good correlation with the seismic response. Thus, one can use the base-case or median/mean model to accurately estimate the median/mean response of dam samples instead of performing computer-intensive IDA with LHS without confusion.

Moreover, based on the mean/median of the IDA curves, damage levels can be defined as follows: (1) local sliding instability with the shearing slide of $40 \mathrm{~mm}$ on the main sliding surface and less than $40 \%$ drainage hole diameter and (2) overall sliding instability of the concrete gravity dam. According to the damage levels defined, seismic fragility curves are obtained. The probability of the occurrence of the 
local sliding damage of the gravity dam is less than $10 \%$, and the probability of reaching the overall sliding instability is almost zero under MDE. The concrete gravity dam is sufficient to maintain good sliding stability even under MCE.

Although the model chosen is only one specific concrete gravity dam without consideration of all the uncertainties and nonlinearities, the conclusions obtained in this paper are of great significance and can serve as a reference for further study. And the further study could be performed considering the following aspects: (1) record-to-record uncertainty, (2) the correlation between different sliding parameters, and (3) material nonlinearity.

\section{Data Availability}

All data generated or analyzed during this study are included in this article.

\section{Conflicts of Interest}

The authors declare that they have no conflicts of interest.

\section{Acknowledgments}

This study was supported by the National Key Research and Development Program (grant no. 2016YFC0401807) and National Natural Science Foundation (grant no. 51709283).

\section{References}

[1] J. Z. Pan, "Design of gravity dam (China: water conservancy and electric power press)," in Chinese, 1987.

[2] National Energy Administration, Concrete Gravity Dam Design Code, (NEA) National Energy Administration, China, NB/T 35026-2014, 1995.

[3] F. O. Hoffman and J. S. Hammonds, "Propagation of uncertainty in risk assessments: the need to distinguish between uncertainty due to lack of knowledge and uncertainty due to variability," Risk Analysis, vol. 14, no. 5, pp. 707-712, 1994.

[4] S. Ferson and L. R. Ginzburg, "Different methods are needed to propagate ignorance and variability," Reliability Engineering and System Safety, vol. 54, no. 2-3, pp. 133-144, 1996.

[5] A. D. Kiureghian and O. Ditlevsen, "Aleatory or epistemic? does it matter?” Structural Safety, vol. 31, no. 2, pp. 105-112, 2009.

[6] M. Leclerc, P. Leger, and R. Tinawi, CADAM User's Manual, Department of Civil, Geological and Mining Engineering, Ecole Polytechnique de Montreal, Quebec, 2001.

[7] P. B. Tekie and B. R. Ellingwood, "Seismic fragility assessment of concrete gravity dams," Earthquake Engineering and Structural Dynamics, vol. 32, no. 14, pp. 2221-2240, 2003.

[8] A. M. Awruch, "Probabilistic finite element analysis of concrete gravity dams," Advances in Engineering Software, vol. 29, pp. 97-104, 1998.

[9] D. Vamvatsikos and C. A. Cornell, "Incremental dynamic analysis," Earthquake Engineering \& Structural Dynamics, vol. 31, no. 3, pp. 491-514, 2002.

[10] J. E. Padgett and R. Desroches, "Sensitivity of seismic response and fragility to parameter uncertainty," Journal of Structural Engineering, vol. 133, no. 12, pp. 1710-1718, 2007.

[11] A. B. Liel, C. B. Haselton, G. G. Deierlein, and J. W. Baker, "Incorporating modeling uncertainties in the assessment of seismic collapse risk of buildings," Structural Safety, vol. 31, no. 2, pp. 197-211, 2009.

[12] M. Dolsek, "Incremental dynamic analysis with consideration of modeling uncertainties," Earthquake Engineering and Structural Dynamics, vol. 38, no. 6, pp. 805-825, 2009.

[13] D. Vamvatsikos and M. Fragiadakis, "Incremental dynamic analysis for estimating seismic performance sensitivity and uncertainty," Earthquake Engineering and Structural Dynamics, vol. 39, no. 2, pp. 141-163, 2010.

[14] M. Alembagheri and M. Ghaemian, "Seismic assessment of concrete gravity dams using capacity estimation and damage indexes," Earthquake Engineering and Structural Dynamics, vol. 42, no. 1, pp. 123-144, 2013.

[15] C. Bernier, J. E. Padgett, J. Proulx, and P. Paultre, "Seismic fragility of concrete gravity dams with spatial variation of angle of friction: case study," Journal of Structural Engineering, vol. 142, no. 5, pp. 1-11, Article ID 05015002, 2016.

[16] B. F. Soysal, B. Binici, and Y. Arici, "Investigation of the relationship of seismic intensity measures and the accumulation of damage on concrete gravity dams using incremental dynamic analysis," Earthquake Engineering and Structural Dynamics, vol. 45, no. 5, pp. 719-737, 2016.

[17] M. Alembagheri and M. Seyedkazemi, "Seismic performance sensitivity and uncertainty analysis of gravity dams," Earthquake Engineering and Structural Dynamics, vol. 44, no. 1, pp. 41-58, 2015.

[18] H. Liang, S. Guo, J. Tu et al., "Seismic stability sensitivity and uncertainty analysis of a high arch dam-foundation system," International Journal of Structural Stability and Dynamics, vol. 19, no. 6, pp. 1950066-1950071, 2019.

[19] A. Morales-Torres, I. Escuder-Bueno, L. Altarejos-García, and A. Serrano-Lombillo, "Building fragility curves of sliding failure of concrete gravity dams integrating natural and epistemic uncertainties," Engineering Structures, vol. 125, pp. 227-235, 2016.

[20] H. M. Westergaard, "Water pressure on dams during earthquakes," Transactions of the American Society of Civil Engineers, vol. 98, pp. 418-472, 1933.

[21] H. Shen, "Performance-based seismic damage analysis and risk assessment of concrete dam-foundation system," Ph.D. thesis in Chinese, Tsinghua University, Beijing, China, 2007.

[22] M. A. Hariri-Ardebili and V. E. Saouma, "Sensitivity and uncertainty quantification of the cohesive crack model," Engineering Fracture Mechanics, vol. 155, pp. 18-35, 2016.

[23] J. Xu, W. Huang, and Y. F. Gao, "International code for design and specification of concrete gravity dam: sub coefficient determination and experimental design," International Conference on Dam Technology and Long-Term Performance, pp. 30-35, 2011, in Chinese.

[24] R. L. Iman and W. J. Conover, "A distribution-free approach to inducing rank correlation among input variables," Communications in Statistics-Simulation and Computation, vol. 11, no. 3, pp. 311-334, 1982. 